\title{
INFLUENCE OF BROWNIAN DIFFUSION ON THE STATICS OF MAGNETIC FLUID
}

\author{
V.G.Bashtovoi ${ }^{1}$, V.K.Polevikov' ${ }^{2}$,A.E.Suprun ${ }^{3}$, \\ A.V.Stroots ${ }^{3}$, S.A.Beresnev ${ }^{2}$ \\ 1 Belarusian National Technical University, \\ 65 Nezavisimosti Ave., Minsk 220013, Belarus \\ 2 Belarusian State University, Minsk 220000, Belarus \\ ${ }^{3}$ Ferrolabs, Inc., 21525 Ridgetop Circle, Suite 260, Dulles VA 20166, USA
}

In the present study, the influence of Brownian diffusion of magnetic particles in a magnetic fluid on a force, which acts upon a fluid volume under an external inhomogeneous magnetic field to be induced by a pair of flat poles, is considered in plane geometry. It is shown that the re-distribution of magnetic particle concentration causes this force to undergo considerable changes with time.

Introduction. As a magnetic fluid represents a colloid of a solid ferromagnetic, it is natural that the processes of magnetophoresis and Brownian diffusion proceed in it [1]. A more detailed description of these processes is given in [2], especially as applied to magnetic concentration convection. They take on great significance when magnetic fluids are used for designing high-precision sensor facilities and their stable parameters should be provided, on the one hand, and when magnetic fields have large gradients, on the other $[3,4]$. The most essential manifestation of the processes of magnetodiffusion is revealed in magnetic fluid seals $[5,6]$.

More frequently, there is a tendency to diminish the influence of these processes in a magnetic fluid by choosing moderate values of magnetic field gradients and also their short residence in the static state in these fields. Nevertheless, these processes always occur in magnetic fluids and their detailed study is of undoubted interest.

When the intensity of a magnetic field is non-linearly distributed in a magnetic fluid, magnetic particles are concentrated in those regions, where the magnetic field intensity is larger. Accordingly, the fluid magnetization increases in these regions. As a rule, these regions are characterized by the highest gradients of the magnetic field intensity. Since a magnetic force acting upon a magnetic fluid volume is defined as the product of fluid magnetization by field gradient, its nonlinear growth with time must be observed. For short, this force will be further referred to as the magnetic weight of a fluid.

1. Governing equations for diffusion of magnetic particles in a magnetic fluid. In the subsequent discussion, the classical theory for diffusion of Brownian particles in the mass force field [7] is applied to magnetic fluids (magnetic colloids). For Brownian particles with a density $\rho$, whose bulk concentration is $C^{\prime}$, the mass conservation law for diffusion of magnetic particles is of the form:

$$
\rho \frac{\partial C^{\prime}}{\partial t}+\operatorname{divi}=0,
$$


where the mass flux density of particles $\mathbf{i}$ is equal to

$$
\mathbf{i}=-\rho D \nabla C^{\prime}+\rho C^{\prime} b \mathbf{f} .
$$

Here $D$ is the diffusion coefficient and $b$ is the mobility of particles. The mobility of particles and the diffusion coefficient are connected by Einstein's relation $D=k T b$. A force $\mathbf{f}$, which acts upon each individual particle of ferromagnetic with a magnetic moment $m_{\mathrm{m}}$ under an inhomogeneous magnetic field with an intensity $H$ and with an intensity gradient $\nabla H$, is defined from the time-averaged value of the projection of this moment $\bar{m}_{\mathrm{m}}$ onto the field direction and is described by the expression $\mathbf{f}=\mu_{0} \bar{m}_{\mathrm{m}} \nabla H$, where $\mu_{0}=1.26 \cdot 10^{-6} \mathrm{H} / \mathrm{m}$ is the magnetic permeability of vacuum. The quantity $\bar{m}_{\mathrm{m}}$ is equal to the product of the particle magnetic moment and the Langevin function $\Lambda: \bar{m}_{\mathrm{m}}=m_{\mathrm{m}} \Lambda(\xi), \Lambda(\xi)=\operatorname{coth} \xi-1 / \xi$, $\xi=\mu_{0} m_{\mathrm{m}} H / k T$, where $k=1.38 \cdot 10^{-23} \mathrm{~J} / \mathrm{K}$ is the Boltzmann constant, $T$ is the absolute temperature [1]. Then $\mathbf{f}=\mu_{0} m_{\mathrm{m}} \Lambda \nabla H$. With regard to this expression, we have:

$$
\mathbf{i}=-\rho D \nabla C^{\prime}+\rho b \mu_{0} m_{\mathrm{m}} C^{\prime} \Lambda \nabla H .
$$

Substitution of Eq. (2) into Eq. (1) yields the following equation for mass transfer in a magnetic fluid:

$$
\frac{\partial C^{\prime}}{\partial t}=D \Delta C^{\prime}-b \mu_{0} m_{\mathrm{m}}\left[\Lambda\left(\nabla C^{\prime} \nabla H+C^{\prime} \Delta H\right)+C^{\prime} \nabla \Lambda \nabla H\right] .
$$

Here $\Delta=\operatorname{div}(\nabla)$ is the Laplace operator.

The condition for impermeability of boundaries, when the normal component of the mass flux density of particles equals zero $\left(i_{n}=0\right)$, is taken as a boundary condition and the initial distribution of magnetic particle concentration is assumed constant, $C^{\prime}(t=0)=\bar{C}$. Moreover, the condition for a constant number of particles in a considered volume

$$
\frac{1}{V} \int_{V_{0}} C^{\prime} \mathrm{d} V=\bar{C},
$$

should be satisfied.

Having chosen as scales a characteristic dimension $l$ for coordinates, $l^{2} / D$ for time, $\bar{C}$ for concentration, and an intensity $H_{0}$ for the magnetic field and having introduced the dimensionless variables: $x_{i}^{\prime}=x_{i} / l, t^{\prime}=t D / l^{2}, C=C^{\prime} / \bar{C}$, $H^{\prime}=H / H_{0}, V^{\prime}=V / h^{3}$, the governing system of equations can be simplified to the following dimensionless form

$$
\begin{aligned}
\frac{\partial C}{\partial t} & =\Delta C-U[\Lambda(\nabla C \nabla H+C \Delta H)+C \nabla \Lambda \nabla H], \\
\Lambda(H) & =\left[\operatorname{coth}(U H)-(U H)^{-1}\right], \\
-\frac{\partial C}{\partial n}+U C \Lambda \frac{\partial H}{\partial n} & =0 \quad \text { (at the impermeable boundaries), } \\
C\left(x_{i}, t=0\right) & =1 \quad \text { (at the initial time moment). }
\end{aligned}
$$

Here $n$ denotes the normal to the boundary. For short, the dimensionless quantities have no primes. A dimensionless criterion $U=\mu_{0} m_{\mathrm{m}} H_{0} / k T$ represents the ratio of the potential energy of a particle in a magnetic field to its thermal energy. 
2. Possibility of equilibrium of magnetic fluid with a non-uniform concentration distribution. As it was mentioned above, a special free convection (magnetic concentration convection) can take place in magnetic fluid with a non-uniform concentration distribution of particles in a non-uniform magnetic field [2]. In most cases, the existence of such convection needs special boundary conditions for the particles' concentration. In this paper, the magnetic fluid is supposed to be unmoved in mechanical equilibrium. It is well known that the condition of fluid equilibrium is the potentiality of the volume force acting in the fluid. For the magnetic force it requires $\operatorname{rot}(M \nabla H)=0$, or collinearity of magnetization $M$ and magnetic field $H$ gradients: $\nabla M \| \nabla H$. As $M=M(C, H)$, this condition is equivalent to the collinearity of concentration $C$ and magnetic field $H$ gradients: $\nabla C \| \nabla H$. It is not difficult to find that if the magnetic fluid is bounded by impermeable immovable solid boundaries at a constant temperature, on which the normal component of concentration flux density of particles equals zero $\left(i_{n}=0\right)$, stationary solutions of all hydrodynamic and heat and mass transfer equations are: 1) the fluid velocity equals zero in the whole volume of the fluid, 2) the fluid temperature is constant, 3 ) the mass flux density of particles equals zero in the whole fluid volume, $\mathbf{i}=0$.

Taking into account that the mass flux density of particles is given by the following expression

$$
\mathbf{i}=-\rho D \nabla C^{\prime}+\rho b \mu_{0} m_{\mathrm{m}} C^{\prime} \Lambda \nabla H,
$$

one can conclude that in this case $(\mathbf{i}=0)$ the stationary distribution of concentration is such that the concentration gradient everywhere is parallel to the gradient of magnetic field intensity: $\nabla C \| \nabla H$. This fact automatically provides the potentiality of volume magnetic force in the equation of magnetic fluid motion.

From the physical view point it means that the re-distribution of magnetic particles in the magnetic fluid under the influence of stationary non-uniform magnetic field takes place in such manner that particles move in the direction of the magnetic field gradient and their steady distribution corresponds to the condition $\nabla C \| \nabla H$ at all points of the fluid volume, i.e., the stable motionless state of the fluid.

It can be noted that in a non-stationary situation $\mathbf{i}=0$ is not the solution of mass transfer equation (1). It means that the non-stationary process of particle concentration re-distribution in the magnetic fluid can be accompanied by a corresponding convection. However, this convection can be regarded as a secondary process relating magnetophoresis and Brownian diffusion and in many cases can be neglected.

3. The magnetic weight of the fluid. Consider the influence of a non-uniform distribution of particle concentration in a magnetic fluid with a magnetization $M$ on a force $\mathbf{F}$ that acts upon its volume $V$ under an inhomogeneous magnetic field. This force can be called a magnetic weight of the fluid and is defined from the expression

$$
\mathbf{F}=\int_{V} \mu_{o} M \nabla H \mathrm{~d} V .
$$

If $M_{\mathrm{fs}}$ is the saturation magnetization of ferromagnetic particles, then the fluid magnetization is determined by the expression $M=C^{\prime} M_{\mathrm{fs}} \Lambda$. If the saturation magnetization of a magnetic fluid is defined as the product of $M_{\mathrm{fs}}$ and the mean bulk concentration of particles $M_{s}=\bar{C} M_{\mathrm{fs}}, M=M_{\mathrm{s}} C \Lambda$, where $C=C^{\prime} / \bar{C}$ has 
the meaning of a relative concentration of particles. Thus, the volume magnetic force in the fluid is defined by the following integral

$$
\mathbf{F}=\int_{V} \mu_{0} M_{\mathrm{s}} C \Lambda \nabla H \mathrm{~d} V
$$

and depends evidently on the particle distribution in the fluid that must be found by solving the system of Eqs. (4).

From this expression it follows that if the magnetic fluid is under saturation $(\Lambda=1)$ and the gradient of the magnetic field intensity is constant and can be taken outside the integral sign, then

$$
\mathbf{F}=\mu_{0} M_{\mathrm{s}} \nabla H \int_{V} C \mathrm{~d} V=\mu_{0} M_{\mathrm{s}} C(\nabla H) V .
$$

i.e., in the magnetic field with a constant gradient the magnetic weight of the fluid in the state of saturation remains constant irrespective of the particle concentration distribution in it.

A different situation arises with a non-linear distribution of the magnetic field intensity in the fluid as well as with the dependence of the fluid magnetization on the magnetic field.

4. Steady one-dimensional solution. Let a layer of a magnetic fluid lie between two impermeable plane-parallel plates distanced as far as $l$, with a magnetic field varying only along the $z$-axis perpendicular to the plates, $H=H(z)$. The vector of the mass flux density of particles $\mathbf{i}$ in this case has only a $z$-projection. Accordingly, in this situation $(\partial C / \partial t=0)$, the first integral of diffusion equation (1) is represented by $i=$ const. Taking into account that this vector is zero at the layer boundaries, the integral equals zero in the entire fluid volume. i.e., from Eqs. (4) we have in the dimensionless form

$$
\frac{\partial C}{\partial z}=U C \Lambda(H) \frac{\partial H}{\partial z} .
$$

Solving this equation yields the following steady distribution of the particle concentration in the considered layer of magnetic fluid

$$
C=\frac{\operatorname{sh}(U H)}{H}\left[\int_{0}^{1} \frac{\operatorname{sh}(U H)}{H} \mathrm{~d} z\right]^{-1}, \quad \int_{0}^{1} C \mathrm{~d} z=1 .
$$

A dimensionless magnetic weight $P$ of the fluid volume with surface $S$ in the longitudinal section is defined as $P=F / \mu_{0} M_{\mathrm{s}} H_{0} S$. From Eq. (5) with regard to Eq. (6), this weight is equal to

$$
P=\int_{0}^{1} C \Lambda \frac{\partial H}{\partial z} \mathrm{~d} z=\frac{1}{U}[C(1)-C(0)] .
$$

If a mean magnetic weight $\bar{P}$ of the fluid is defined as its value at a relative particle concentration equal to unity (homogeneous fluid), then

$$
\bar{P}=\int_{0}^{1} \Lambda(H) \frac{\partial H}{\partial z} \mathrm{~d} z=\frac{1}{U} \int_{0}^{1} \frac{\mathrm{d} C}{C}=\frac{1}{U} \ln \frac{C(1)}{C(0)} .
$$




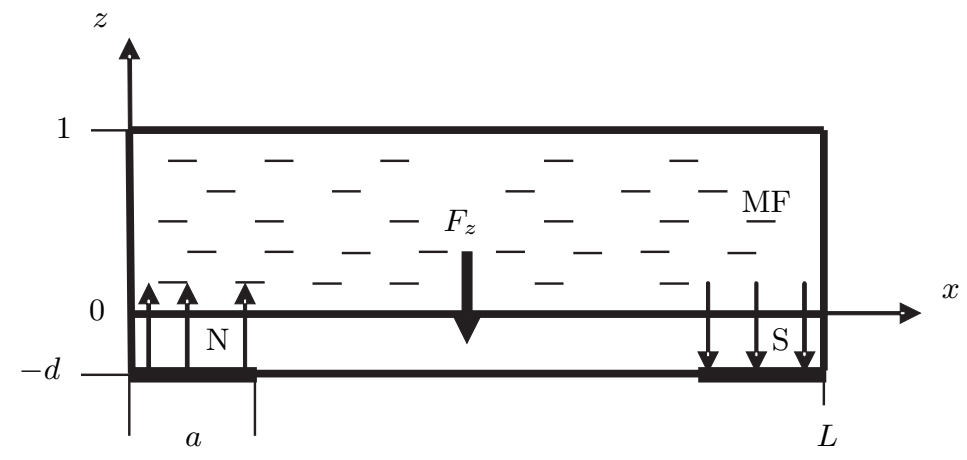

Fig. 1. Geometry of the problem.

It is convenient to analyze further a relative value of this weight

$$
P_{r}=P / \bar{P}=[C(1)-C(0)] /[\ln C(1)-\ln C(0)] .
$$

For large values of the magnetic field intensity $(U \gg 1)$, when the fluid can be assumed saturated and $\Lambda=1$, solving Eq. (8) for the above-defined values yields the following equations

$$
\begin{aligned}
& C=\exp (U H) / \int_{0}^{1} \exp (U H) \mathrm{d} z, \quad P=[C(1)-C(0)] / U, \\
& \bar{P}=[\ln C(1)-\ln C(0)] / U=H(1)-H(0) .
\end{aligned}
$$

5. Time-dependent two-dimensional problem. A volume of the magnetic fluid filling a rectangular cavity with solid walls $0 \leq z \leq 1,0 \leq x \leq L$ is considered (Fig. 1). The cavity height is chosen as the length scale.

A magnetic field is induced by two opposite poles $N, S$ of a magnetic field source that are located parallel to the cavity boundary $z=0$ at its extreme points $x=0$ and $x=L$ and at a distance $d$ from it. The pole width is $a$ and the magnetic flow through each of them per unit length in the transverse direction is $\Phi_{0}=\int_{0}^{a} H(z=-d) \mathrm{d} x . H_{0}=\Phi_{0} / a$ is accordingly taken as the scale of the magnetic field intensity. The problem is considered when the magnetic field perturbations caused by the presence of magnetic fluid are neglected. For the lines of magnetic force $\psi(x, z)\left(H_{x}=\partial \Psi / \partial z, H_{z}=-\partial \Psi / \partial x\right)$ the Laplace equation

$$
\Delta \psi=\frac{\partial^{2} \psi}{\partial x^{2}}+\frac{\partial^{2} \psi}{\partial z^{2}}=0
$$

is solved with the following boundary conditions at $z=-d$ :

$$
\begin{array}{ll}
\text { over the section } & 0 \leq x \leq a: \partial \Psi / \partial z=0, \\
& \int(\partial \Psi / \partial x) \mathrm{d} x=a, \psi_{1}(a)-\psi(0)=a, \\
& \\
\text { over the section } & a \leq x \leq L-a: \partial \Psi / \partial x=0, \psi=\text { const }=a, \\
\text { over the section } \quad & L-a \leq x \leq L: \partial \Psi / \partial z=0, \\
& \int(\partial \Psi / \partial x) \mathrm{d} x=-a, \psi(L)-\psi(L-a)=-a, \\
& \frac{\partial \psi}{\partial z}=0, \frac{\partial \psi}{\partial x}=0, \psi=\text { const }=0 .
\end{array}
$$


After the configuration of the magnetic field

$$
H=\sqrt{(\partial \Psi / \partial x)^{2}+(\partial \Psi / \partial z)^{2}}
$$

is calculated, the distribution of a relative particle concentration in the cavity is found numerically by solving the set of Eqs. (4) in a two-dimensional approach. The time explicit difference scheme and the alternating direction method were used.

With the above approach, the resulting magnetic force acting upon the investigated fluid volume (magnetic weight) has only a $z$-projection and is found using the considered area integral:

$$
P=F_{z}=\int_{0}^{L} \int_{0}^{1} C \Lambda(\partial H / \partial z) \mathrm{d} x \mathrm{~d} z .
$$

As in Sec. 3, the mean magnetic weight is defined as

$$
\bar{P}=P(C=1)=\int_{0}^{L} \int_{0}^{1} \Lambda(\partial H / \partial z) \mathrm{d} x \mathrm{~d} z
$$

and the relative weight as $P_{r}=P / \bar{P}$.

6. Results and discussion. First of all, it should be noted that the above-described processes can essentially manifest themselves at the values of the magnetic parameter $U$ larger than unity. At the value of the magnetic moment of particles $m_{m}=2.5 \cdot 10^{-19} \mathrm{~J} / \mathrm{T}$ typical of magnetic fluids and at room temperature $T=300 \mathrm{~K}$, the quantity $U=1$ is valid at a magnetic field intensity of about 10 $\mathrm{kA} / \mathrm{m}$. In the gaps of magnetic fluid seals, dampers and miniature sensors, where high gradient magnetic fields are induced, the values of this parameter can attain several unities and even dozens. In fact, when these processes are considered, it

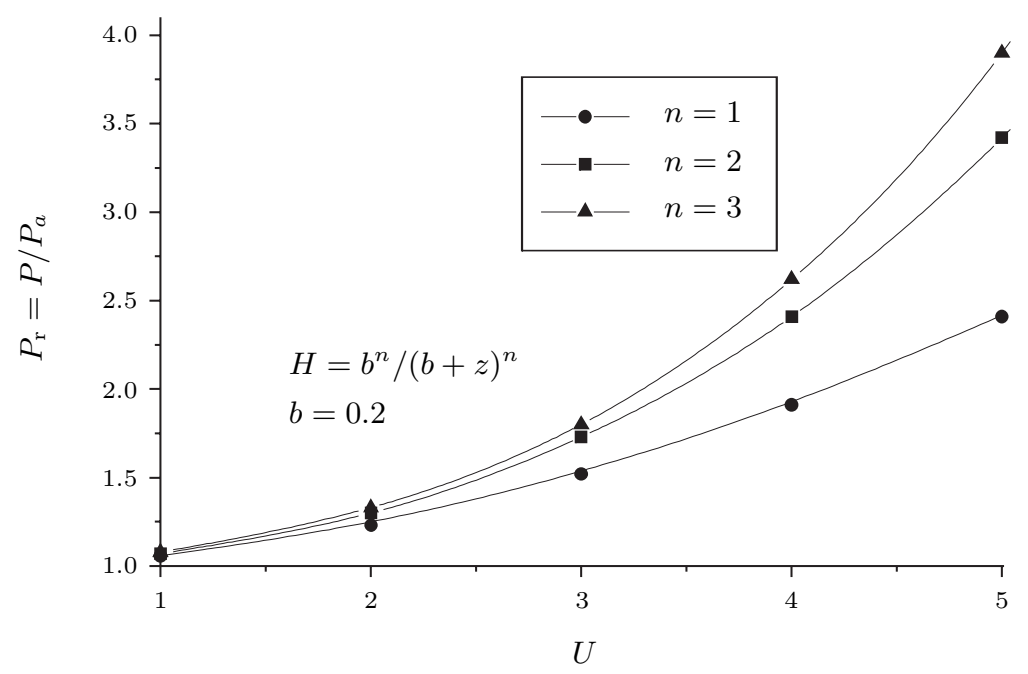

Fig. 2. Relative magnetic weight $P_{r}$ of the fluid vs. the magnetic number $U$ in the one-dimensional problem approximation. 


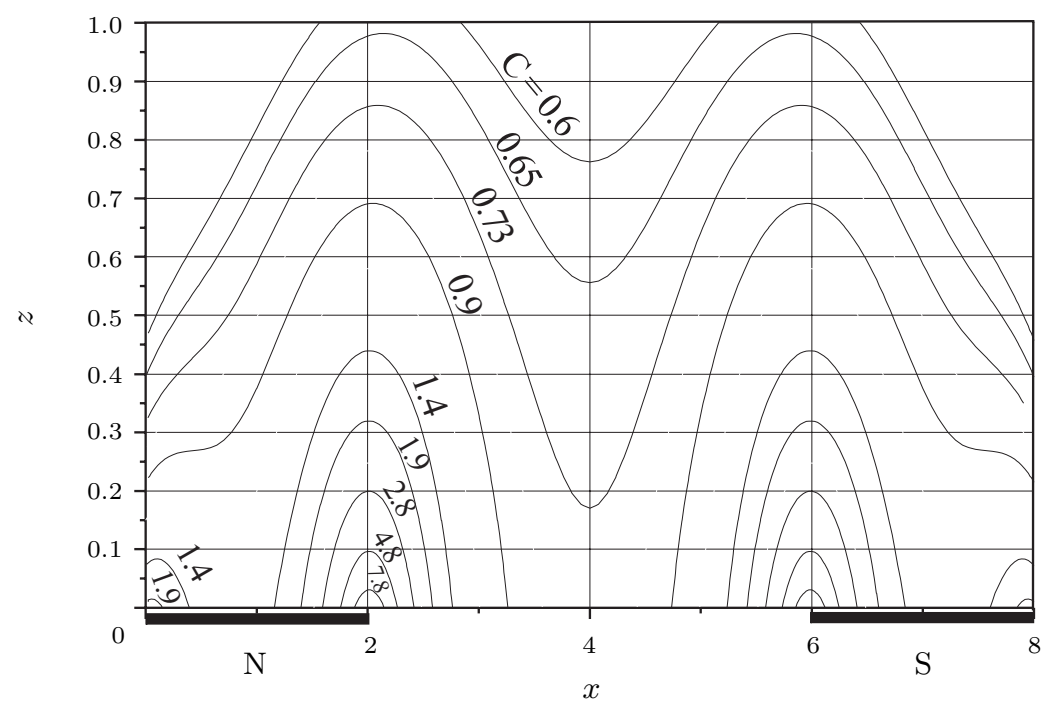

Fig. 3. Isolines of the steady-state relative concentration $C=$ const at $U=6$.

should be borne in mind that irreversible concentration changes in the fluid can appear under strong magnetic fields. In particular, irreversible sedimentation of particles at magnet surfaces is possible. Owing to this, the below analysis of the obtained results will be limited by the value of relative particle concentration equal to 5 or by the condition that regions with a larger concentration occupy no more than $5 \%$ of the magnetic fluid volume.

The plots in Fig. 2 demonstrate the influence of Brownian diffusion on the magnetic weight of the fluid in the one-dimensional problem approximation. Calculations are made using Eqs. (7) and (8) for the following model configuration of the dimensionless magnetic field intensity: $H=b^{n} /(b+z)^{n}$. The change in power $n$ enables one to follow the influence of the degree of magnetic field non-linearity on the analyzed processes. The condition $H(z=0)=1$ means that the scale of the magnetic field intensity $H_{0}$ is represented by its value at the point $z=0$.

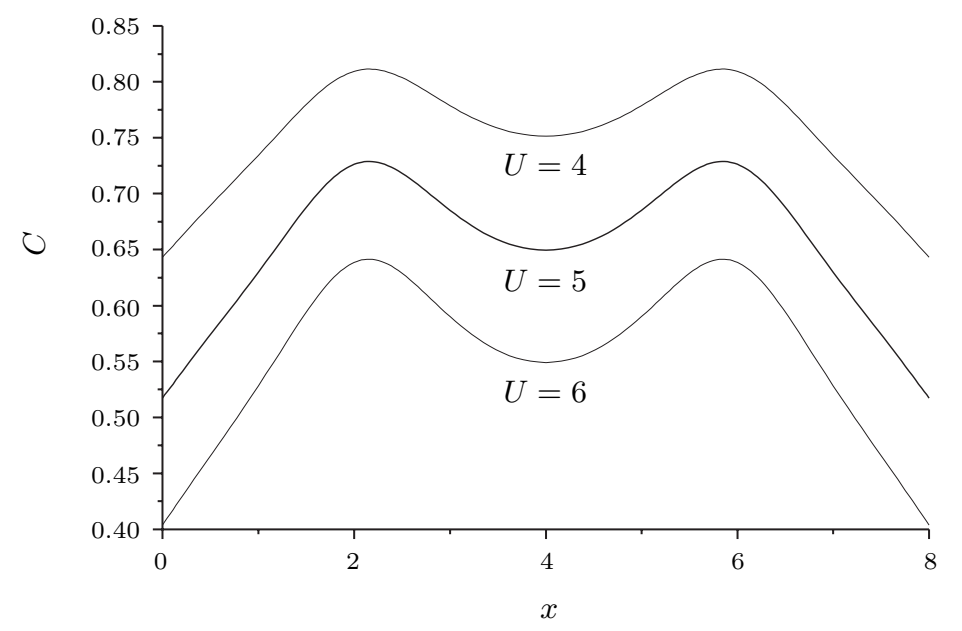

Fig. 4. Distribution of the relative steady-state particle concentration $C$ at the upper boundary of the cavity for $z=1$ at different values of the magnetic parameter $U$. 


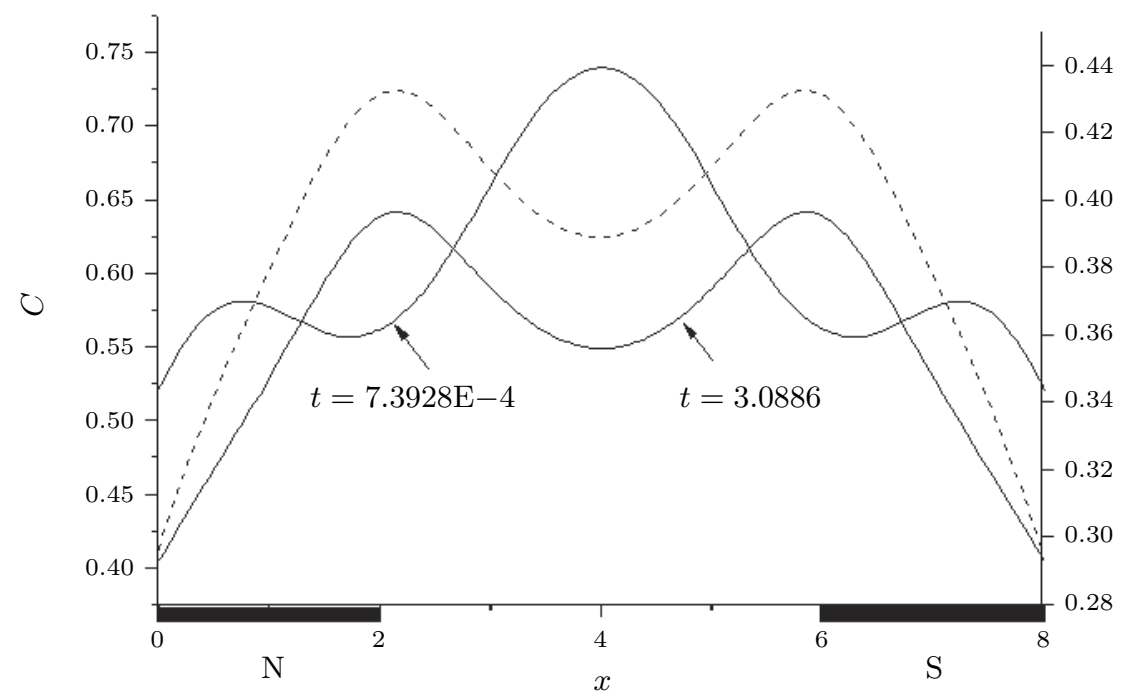

Fig. 5. Distribution of the relative particle concentration $C$ at different time moments (solid lines) and of magnetic field intensity $H$ (dotted line) at the upper boundary of the cavity for $z=1$ at $U=6$.

It is seen that the magnetic weight of the fluid can increase in several times with the increase of $U$. This increase occurs the faster the higher are the gradients of the magnetic field intensity.

Figs. 3-6 show the results of numerical computations at $a=2, L=8, d=0.27$ in the two-dimensional approximation.

Fig. 3 sketches the steady-state field of the particle concentration, the isolines of which qualitatively correspond to those of magnetic field intensity. The field distribution at the upper boundary of the cavity is shown in Fig. 5.

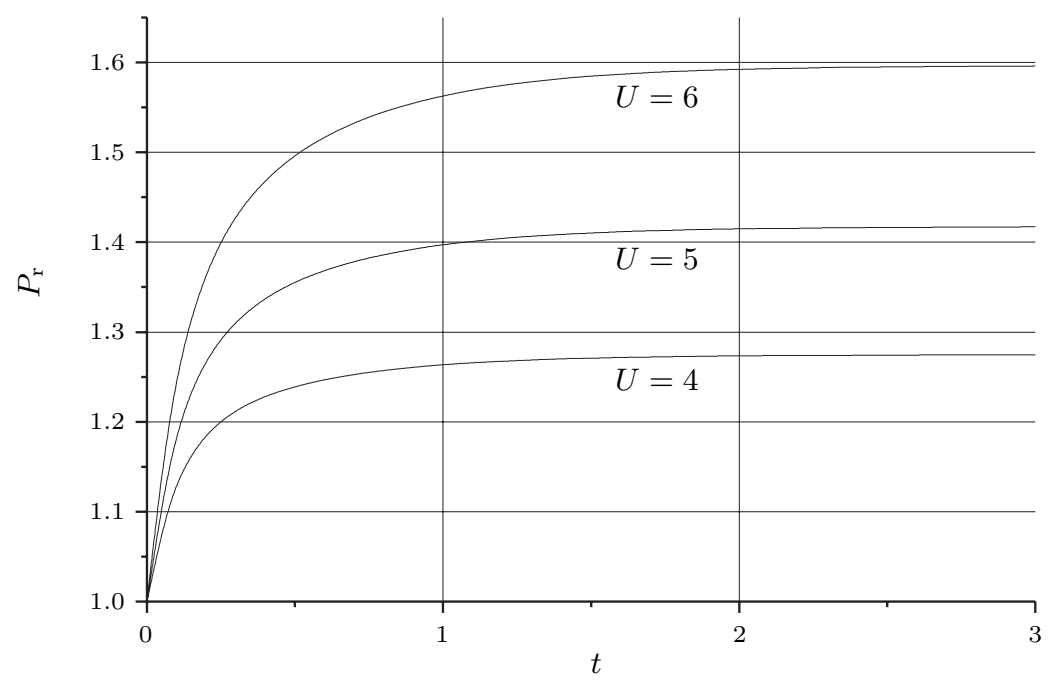

Fig. 6. Time-variations of the fluid relative magnetic weight at different values of the magnetic parameter $U$. 
The particle concentration is larger in those places, where the magnetic field intensity is higher. As this takes place, the regions with an anomalously large concentration $(C>5)$ occupy a reasonably small space near the inner edges of the poles, where the changes in the magnetic field are the widest. Such behaviour is attibuted to the steady-state distribution of the particle concentration at the upper boundary of the cavity, as shown in Fig. 4. At this boundary, the values of the relative particle concentration are much smaller than unity already at $U>2$ that is due to a large flow of particles from it to the magnet poles.

Of interest are the time-variations of the particle concentration distribution at the upper boundary of the cavity, which are illustrated by the plots in Fig. 5 . At the initial time instants, this distribution in its character is in direct opposition to the steady-state one. With time, the minima in the places of highest magnetic field intensity are replaced by maxima, and a maximum in the central region of the cavity is replaced by a minimum. This is due to the fact that during the initial time period, the $z$-components of the magnetic field intensity gradients are dominant, and the motion of particles directly to the poles (along the $z$-axis) is the stongest. Then in due course, the flow of particles develops in the longitudinal direction (along the $x$-axis) towards the central region of the cavity, and the particle concentration increases in these regions.

The above-described re-distribution of the particle concentration causes the magnetic weight of the fluid in the considered cavity to essentially increase with time (Fig. 6). This change is most efficient within the time interval of 0 to 0.25 . Then the weight of the fluid reaches its steady value. It is natural that the influence of these processes is the more pronounced the larger is the value of the magnetic parameter $U$.

In conclusion it can be noted that more precise non-stationary results can be obtained in future taking into account the magnetic concentration convection.

\section{REFERENCES}

[1] R.E. Rosensweig. Ferrohydrodynamics (Cambridge University Press, New York, 1985).

[2] E. Blums, A. Cebers, M.M. Maiorov. Magnetic Fluids (W. de Gryuter, Berlin, 1997).

[3] Magnetic Fluids and Applications. Handbook, Eds. B. Berkovski, V. Bashtovoi (Begell House, Inc. Publishers, New York, 1996).

[4] A.E. Suprun, D.V.Simonenko, Yu.I. Romanov. US Patent 6,731,268, 2004.

[5] S. TAKeтOMi. Motion of ferrite particles under a high gradient magnetic field in a magnetic fluid shaft seal. Jap. J. Appl. Phys., vol. 19 (1980), no. 10, pp. 1929-1936.

[6] I. Anton, L. Vekash, I. Potents, D. Bika. Behavior of magnetic liquids in an inhomogeneous magnetic field. Magnetohydrodynamics, vol. 21 (1985), no. 3, pp. 213-218.

[7] L.D. Landau, E.M. Lifshitz. Fluid Mechanics (Pergamon Press, London, 1959).

Received 09.11.2006 